\title{
Re: Caiulo VA, Gargani L, Caiulo S, Fisicaro A, Moramarco F, Latini G, Picano E. Lung ultrasound in bronchiolitis: comparison with chest X-ray. Eur J Pediatr. 2011;170: 1427-33
}

\author{
Daniela Catalano $\cdot$ Marco Sperandeo $\cdot$ Guglielmo Trovato
}

Received: 23 July 2013 / Accepted: 26 September 2013 /Published online: 13 October 2013

(C) Springer-Verlag Berlin Heidelberg 2013

Dear Editor,

The comment of Toma [5] to the article by Caiulo et al. [1] appropriately raises serious concerns in their recommendation regarding lung ultrasound in infant's bronchiolitis. We too disagree with Caiulo et al. [1] because the Authors state that from their data "correlation between clinical and lung ultrasound findings and correlation between clinical and CXR findings are demonstrated" [1]. This is not evidence-based; the methods used (no detail on equipment setting, no rationale for the chest $\mathrm{x}$-ray prescription) and the published figure are not demonstrating any straightforward information, no convincing image is provided, and no correlation was performed. How authors [1] can state that their data show that LUS may help to better characterize and stratify the patients for the follow-up of lung abnormalities, with sensitivity not inferior to CXR? No sensitivity analysis is quoted in the study. According to the assertion of the authors [1], diagnosis of bronchiolitis is a clinical one; in our daily practice, chest $\mathrm{x}$-ray may help mainly to exclude other different acute pulmonary disease. Which was the indication for $\mathrm{x}$-rays in these seemingly nonconsecutive patients? Moreover, the statement that the lung ultrasound learning curve is simple and fast compared to other ultrasound applications is not the shared experience of teaching centers, as ours, working at postgraduate level. Lung ultrasound procedure and interpretation are not the easiest component of any US curriculum, since it implies the same level of pitfalls of ultrasound assessment of other organs $[2,3]$. Ultrasound procedures are probably among the most useful and specialized skills in clinical ultrasound, requiring imaging quality, sustainability, repeatability, and safety of the procedure [4]. Relying on artifacts, such as B-lines, is not an evidence-based best-practice imaging clue and lacks diagnostic specificity and sensitivity [6]. We warrant that recommendations, included in any article, should be, as always in your Journal, more evidence-based and less conjectural.

\section{References}

1. Caiulo VA, Gargani L, Caiulo S, Fisicaro A, Moramarco F, Latini G, Picano E (2011) Lung ultrasound in bronchiolitis: comparison with chest X-ray. Eur J Pediatr 170:1427-33

2. Sperandeo M, Carnevale V, Muscarella S et al (2011) Clinical application of transthoracic ultrasonography in inpatients with pneumonia. Eur J Clin Invest 41:1-7

3. Sperandeo M, Filabozzi P, Varriale A et al (2008) Role of thoracic ultrasound in the assessment of pleural and pulmonary diseases. J Ultrasound 11:39-46

4. Sperandeo M, Sperandeo G, Varriale A et al (2006) Contrast-enhanced ultrasound (CEUS) for the study of peripheral lung lesions: a preliminary study. Ultrasound Med Biol 32:1467-72

5. Toma P (2013) Lung ultrasound in bronchiolitis. Eur J Pediatr 172:713

6. Trovato GM, Sperandeo M (2013) Sounds, ultrasounds, and artifacts: which clinical role for lung imaging? Am J Respir Crit Care Med 187:780-1

D. Catalano $\cdot$ G. Trovato $(\bowtie)$

Department of Medical and Pediatric Sciences, University of

Catania, Catania, Italy

e-mail: Guglielmotrovato@unict.it

M. Sperandeo

Department of Internal Medicine, IRCCS Casa Sollievo della

Sofferenza Hospital, San Giovanni Rotondo, Italy 